\title{
Ultrasound-guided transversalis fascia plane block provides analgesia for anterior iliac crest bone graft harvesting
}

\author{
Ki Jinn Chin, MBBS • Vincent Chan, MD • \\ Peter Hebbard, MBBS · Jyh Shen Tan, MMed • \\ Meera Harris, MB BChir · Danielle Factor, MBBS
}

Received: 22 August 2011/ Accepted: 7 October 2011/Published online: 19 October 2011

(C) Canadian Anesthesiologists' Society 2011

\section{To the Editor,}

The iliac crest is primarily innervated by the $\mathrm{L} 1$ nerve root via the iliohypogastric and ilioinguinal nerves. We describe our preliminary experience with a transversalis fascia plane (TFP) block, ${ }^{1}$ which targets these nerves in the plane between the transversus abdominis (TA) aponeurosis or muscle and the deeper transversalis fascia. It can thus provide effective analgesia for anterior iliac crest bone graft (ICBG) harvesting. This technique is distinct from the transversus abdominis plane block, which does not reliably block the L1 dermatome, ${ }^{2,3}$ although its use has been described in ICBG harvesting. ${ }^{4}$

Following Ethics Board approval, we reviewed the records of 27 adult patients who underwent harvesting of an anterior ICBG as part of surgery on the distal forearm and wrist between November 1, 2009 and February 28, 2011 at the Toronto Western Hospital. All patients underwent general anesthesia preceded by a single-shot brachial plexus block with $30-40 \mathrm{~mL}$ of local anesthetic (1:1 mixture of $2 \%$ lidocaine and $0.5 \%$ bupivacaine, with epinephrine $2.5 \mu \mathrm{g} \cdot \mathrm{mL}^{-1}$ ). Twelve (44\%) patients also underwent an ultrasound-guided TFP block. The

K. J. Chin, MBBS $(\varangle) \cdot$ V. Chan, MD - M. Harris, MB BChir

Toronto Western Hospital, University of Toronto,

Toronto, ON, Canada

P. Hebbard, MBBS

North East Health, Wangaratta, VIC, Australia

J. S. Tan, MMed

Tan Tock Seng Hospital, Singapore, Singapore

D. Factor, MBBS

Queen Elizabeth Hospital, Woolwich, UK ultrasound probe was placed in a transverse orientation above the iliac crest; and the external oblique, internal oblique (IO), and TA muscles were identified and traced posteriorly until first the TA muscle and then the IO muscle tapered into their common aponeurosis, adjacent to the quadratus lumborum muscle (Figure, A). The tip of a 22-gauge 80-mm block needle was positioned just deep to the TA muscle and its aponeurosis at the point where the TA tapered off. Ropivacaine $0.5 \%(20 \mathrm{~mL})$ with epinephrine $5 \mu \mathrm{g} \cdot \mathrm{mL}^{-1}$ was injected into the plane between the TA and underlying transversalis fascia (Figure, B). Intraoperative and postoperative analgesia of the ICBG harvest site was provided by systemic opioids at the discretion of the operating theatre and postanesthesia care unit (PACU) staff. We extracted data on opioid consumption and pain scores during the immediate perioperative period, defined as the time at which the patient entered the operating room to the time the patient left the PACU. We converted all opioid doses into intravenous morphine equivalents and divided patients into two groups for comparison based on whether they had undergone a TFP block.

The mean (standard deviation) perioperative opioid dose was substantially lower in the patients who underwent a TFP block [18.5 (9.6) $\mathrm{mg}$ of intravenous morphine equivalent] than in those who did not [32.6 (12.4) $\mathrm{mg}$ ] $(P=0.006)$. These patients received less intravenous morphine during both the intraoperative period [10.2 (5.6) $\mathrm{mg}$ vs 17.4 (6.6) $\mathrm{mg} ; P=0.009]$ and the PACU period [8.3 (9.6) $\mathrm{mg} v \mathrm{vs} 15.1(11.2) \mathrm{mg} ; P=0.114]$. It is of note that only one (8\%) patient received intravenous ketorolac $30 \mathrm{mg}$ in the TFP group compared with five (33\%) patients in the other group. Patients who underwent a TFP block also had lower resting pain scores in the PACU. Pain scores (median [range]) were lower in the TFP group both at admission to the PACU (2 [0-6] vs 6 [5-7]; $P=0.015)$ as 


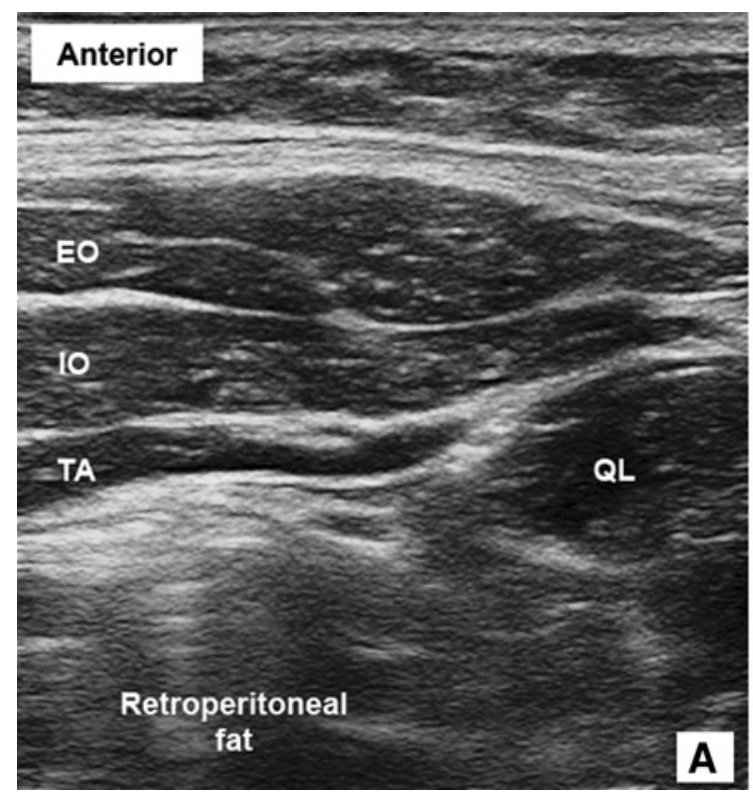

Figure (A) Composite ultrasound image of the internal oblique (IO) and transversus abdominis (TA) tapering off posteriorly into their common aponeurosis adjacent to the quadratus lumborum (QL). (B) The needle tip is positioned just under the aponeurosis of the TA.

well as at discharge from the PACU (0 [0-3] vs 4 [3-4]; $P=0.021$ ).

The results of this small retrospective pilot study suggest that the TFP block, which targets the ilioinguinal and iliohypogastric nerves along the posterior third of the iliac crest and proximal to the origin of their lateral cutaneous branches, ${ }^{5}$ provides effective analgesia for anterior ICBG harvesting, as indicated by the significantly lower perioperative opioid consumption and pain scores during the immediate postoperative period. Further investigation is warranted to verify these findings and to define the clinical characteristics of the block better.

Competing interests None declared.

\section{References}

1. Hebbard PD. Transversalis fascia plane block, a novel ultrasoundguided abdominal wall nerve block. Can J Anesth 2009; 56: 618-20.

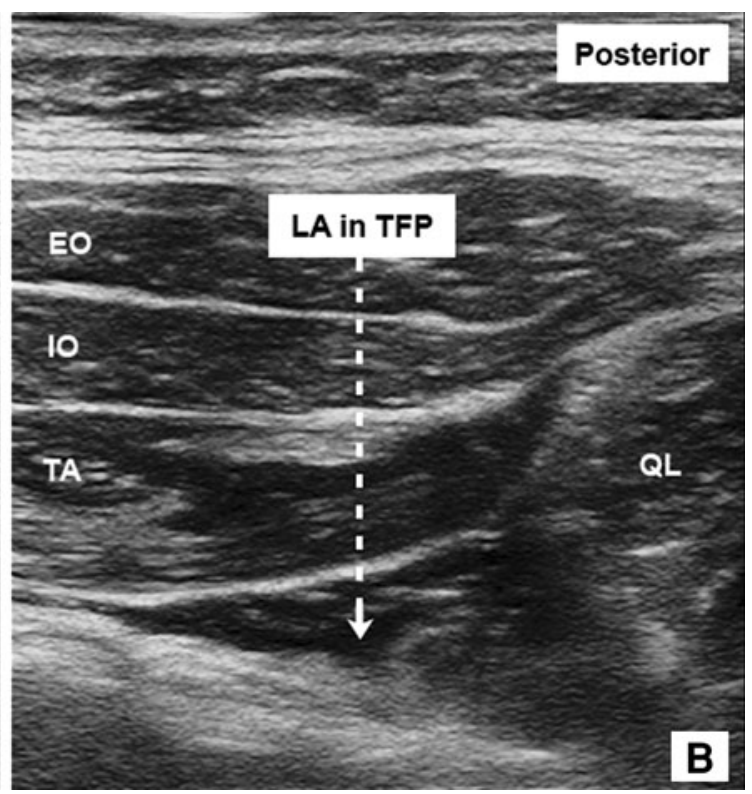

Injection in this location should result in visible local anesthetic (LA) spread in the transversalis fascia plane (TFP). EO = external oblique. (Reproduced with permission from www.usra.ca)

2. Lee TH, Barrington MJ, Tran TM, Wong D, Hebbard PD. Comparison of extent of sensory block following posterior and subcostal approaches to ultrasound-guided transversus abdominis plane block. Anaesth Intensive Care 2010; 38: 452-60.

3. Fredrickson MJ, Paine C, Hamill J. Improved analgesia with the ilioinguinal block compared to the transversus abdominis plane block after pediatric inguinal surgery: a prospective randomized trial. Pediatr Anesth 2010; 20: 1022-7.

4. Chiono J, Bernard N, Bringuier $S$, et al. The ultrasound-guided transversus abdominis plane block for anterior iliac crest bone graft postoperative pain relief: a prospective descriptive study. Reg Anesth Pain Med 2010; 35: 520-4.

5. Jamieson RW, Swigart LL, Anson BJ. Points of parietal perforation of the ilioinguinal and iliohypogastric nerves in relation to optimal sites for local anaesthesia. Q Bull Northwest Univ Med Sch 1952; 26: $22-6$. 